\title{
Factores asociados al consumo de cigarrillos en estudiantes de medicina
}

\author{
Br. Juan José Amador Sánchez, Br. Kester Enrique Barquero Duarte, \\ Br. Alexis Eruben Castellón Méndez, Bra.Larissa Jhadith López Haar. \\ Facultad de Medicina. ${ }^{1}$ \\ Recibido 4 de mayo 2013-Aprobado 28 de octubre 2013.
}

\section{INTRODUCCIÓN}

El tabaco es un estimulante del sistema nervioso central que muchos estudiantes universitarios usan con el fin de relajarse, aun conociendo los efectos nocivos y dañinos que este produce. El consumo de tabaco en los países en desarrollo constituye un problema de salud pública cuya iniciación generalmente ocurre en la adolescencia, donde más de la mitad de los jóvenes al llegar a ser adultos poseen hábito tabáquico. El tabaco es tan adictivo como la droga pura, ya que se trata de un producto psicoactivo que afecta los procesos químicos del cerebro y del sistema nervioso. Además tiene reconocidos efectos nocivos sobre el sistema respiratorio, dañando gravemente bronquios y pulmones, llegando a producir bronquitis crónicas, enfisema y cáncer pulmonar, entre otras. Los fumadores, además de dañar su propia salud, perjudican a los que los rodean. Es notorio que lo que se ha denominado "tabaquismo pasivo" es peligroso para quienes lo sufren, justificándose plenamente las medidas de protección que se han adoptado en los diversos entornos en que se producen estas situaciones. Es necesario destacar que reviste suma gravedad el hecho de que las mujeres fumen durante el embarazo, debido a que pueden llegar a tener partos prematuros, mayor incidencia de abortos, mortalidad perinatal, niños con bajo peso de nacimiento, entre otros problemas.

La falta de estudios sobre esta línea de investigación nos ha motivado la realización de este trabajo, con cuyos resultados se pretende obtener una base de datos que refleje un diagnóstico del consumo de tabaco de la población en estudio. Los beneficiarios directos serán todos los estudiantes de la Facultad de Ciencias Médicas de la UNAN-Managua, y los beneficiarios indirectos todos los estudiantes de la UNANManagua. En este estudio, queremos identificar los factores asociados con el consumo de cigarrillos en los estudiantes del primer al tercer año de la carrera de medicina de la Facultad de Ciencias Médicas de la UNAN-Managua durante el II Semestre 2012, con el fin de sensibilizarlos acerca de los riesgos que trae consigo el hábito de fumar.

\section{MATERIALES Y MÉTODOS}

La investigación realizada es de tipo descriptiva y de corte transversal, pues describe los datos y características del fenómeno en estudio en un periodo determinado de tiempo. Fue elaborada en Universidad Nacional Autónoma de Nicaragua, Recinto Universitario "Rubén Darío", Facultad de Ciencias Médicas.

El universo está constituido por 1034 estudiantes matriculados que cursan de primero a tercer año la Carrera de Medicina en la Facultad de Ciencias Médicas. El método utilizado para la selección de la muestra fue de tipo probabilístico, aleatorio simple, pues todas las alumnos (as) tenían la misma probabilidad de ser seleccionadas. Por tanto, la muestra está constituida por 385 alumnos de primero a

\footnotetext{
${ }^{1}$ Trabajo dirigido por los maestros José Thomas Dávila Arriola y Marilú Cerda Lira.
} 
tercer año de la Carrera de Medicina en la Facultad de Ciencias Médicas de la Universidad Nacional Autónoma de Nicaragua, Managua representando un 37\% del universo. El número de personas a encuestar se obtuvo mediante la fórmula para el cálculo de la muestra para determinar la proporción en un solo grupo.

$\mathrm{n}=[\mathrm{Nx} * \mathrm{Zax} * \mathrm{Px}(1-\mathrm{P})] /(\mathrm{n}-1) * \mathrm{~d} 2$

$=[1034 * 1.962 * 0.5 * 0.5] /(1034-1) *(0.05) 2$

$=993.0536 /(1033)(0.0025)$

$=993.0536 / 2.5825$

$=384.5318$

\section{RESULTADOS Y DISCUSIÓN}

\section{Prevalencia del consumo de tabaco de acuerdo a las características sociodemográficas de los estudiantes.}

La prevalencia del Consumo de cigarrillos es del 17.1\%. Tanto en mujeres como hombres se refleja un alto porcentaje de personas que nunca han fumado (70.9\% y $50.7 \%$, respectivamente), después ambos géneros coinciden que la edad de inicio de fumado que predomina es después de los 16 años, siguiéndole de los 14 a 15 años. Los estudiantes encuestados dentro de las edades de 18 a 19 años de edad representan el grupo con mayor población representando el $52.4 \%$, divididos en $52.8 \%$ de sexo femenino y $47.2 \%$ del sexo masculino, donde se determinó que el índice de fumado es mayor en los hombres con un porcentaje de $24.3 \% \%$, y menor en las mujeres es de $10.8 \%$.

También el estudio resaltó que el hábito de fumar cigarrillos prevalece con mayor significancia en estudiantes que provienen de colegios privados con un $21.3 \%$ en contraste de los que provienen de colegios públicos representados por un $14.2 \%$, con una relevancia de que el año académico en el que más se encontró que los estudiantes fuman más es en segundo año con un $24.4 \%$ seguido de tercer año con un $22.3 \%$ y en menor proporción los estudiantes de primer año representados con $4.7 \%$. Por otra parte se observa que en las hogares en donde ambos padres fumaban, la prevalencia de hijos fumadores es mayor con un $33.3 \%$, seguidos por un descenso al $24.7 \%$ en donde solamente el padre era fumador y para los encuestados quienes no conviven con una persona fumadora, la prevalencia disminuyó a un $15.7 \%$.

\section{Factores asociados al hábito de fumar cigarrillos}

De las personas que posee el hábito de fumar que son un $17.1 \%$ un $38.8 \%$ de estos que representa la mayoría, convive con otras personas que no pertenecen al núcleo familiar. Mientras tanto estos valores disminuyen en los que conviven con ambos padres con un $14.4 \%$ y aún más en los que conviven con uno de los progenitores.

Los encuestados procedentes de centros públicos y privados en su mayoría nunca han fumado (64.3\% y $80 \%$, respectivamente), después los encuestados de ambas procedencias expresan que la edad de inicio de fumado más frecuente es a los 16 años o más, seguido de los 14-15 años, pero en los centros públicos luego predomina la edad de 10 a 11 años, a diferencia de los privados un poco mayor a la edad de 13 años. Esto indica que la procedencia escolar no influye significativamente en la edad de inicio de fumado, pues en ambos casos hay correspondencia con los porcentajes. De los datos obtenidos en las encuestas, los que tienen ingresos menores de 500 consumen en su mayoría de 2-3 cigarrillos diarios o de 4 a 6 cigarrillos (40\% cada uno respectivamente), con ingresos de hasta 1,000 córdobas, la mayoría fuma de 1 a 3 al día, seguido de 7 a 9. Los que tienen ingresos de hasta 1,500 la mayoría fuma de 4-6 cigarrillos. Los encuestados que tienen de entrada de 1,501 a 2,000 la mayoría fuma de 1-3 cigarrillos. Con ingresos de hasta 2,500 el mayor porcentaje corresponde a 4-6 cigarrillos al día, y los que tienen ingresos de 3,000 o 
más la mayoría fuma de 1 a 3 cigarrillos diarios. De esta manera se puede observar que tanto los que tienen los ingresos más bajos o los ingresos más altos consumen la mínima cantidad de cigarrillos al día, mientras que los que tiene ingresos intermedios fuman un poco más de 4-6 cigarrillos diarios.

Se pudo determinar que el consumo de cigarrillos en independiente de la cantidad de los ingresos económicos ya que de las personas con ingresos mayores o igual a 3000 fuma un $28.9 \%$, seguidamente fuma un 5.7\% de los que ganan entre 2501-3000, de los que reciben entre 1501-2000 fuman 21.3\%, en contraste con los que ganan entre 501 a 1000 de los cuales fuma un $20.5 \%$, estos datos reflejan que no hay una gran diferencia de consumo en relación a los ingresos. De los datos válidos obtenidos, se puede apreciar que los alumnos en su mayoría gastan menos de 140 córdobas semanalmente en cigarrillos, no influenciando la cantidad de ingreso económico mensual. Estas cifras se relacionan con los datos anteriores de la cantidad de cigarrillos fumados diarios respecto al ingreso económico.

De los encuestados válidos reflejan que los lugares con mayor frecuencia donde aprendieron a fumar son el colegio, la universidad, casas de amigos y en fiestas y reuniones sociales. Minoritariamente en casa y en otros lugares. De esta forma se puede apreciar la influencia de amistades, círculos sociales en el aprendizaje del mal hábito de fumado. De los encuestados válidos reflejan que los lugares con mayor frecuencia donde aprendieron a fumar son el colegio, la universidad, casas de amigos y en fiestas y reuniones sociales. Minoritariamente en casa y en otros lugares. De esta forma se puede apreciar la influencia de amistades, círculos sociales en el aprendizaje del mal hábito de fumado.

Con respecto a la educación familiar, el hábito de consumo de tabaco se distribuye casi equitativamente en todas las personas independientemente de la cantidad de información recibida por parte de su familia. Se observó que la cantidad de personas que más fuman son las que no consideran que el cigarrillo sea perjudicial para la salud. De los encuestados, fuman más los que están de acuerdo que el consumo de cigarrillos proporciona mayores amistades. Las personas que están de acuerdo que el consumo de cigarrillos les hace sentir más comodidad en reuniones sociales son las que presentan una mayor frecuencia de consumo de tabaco.

Las personas que creen que el consumo de cigarrillos aumenta el atractivo físico son las que representan la mayor cantidad de datos con respecto a los que consumen tabaco. La mayor parte de los que han consumido o consumen cigarrillos es por placer, la segunda razón es por ansiedad, seguido de las personas que lo hace por otra razón (estrés, tristeza...etc.) y los valores más bajo reflejan a los que fuman por sentirse bien con los amigos y los que consumen cigarrillos por problemas familiares. De los encuestados la mayoría expresa que el medio que más implementa campañas antitabaco es la televisión, seguido por otras fuentes (carteles, revistas, películas. etc.) y en tercer lugar indican que ningún medio de comunicación implementa campañas en contra del tabaco. Del 100\% de total de los encuestados en su mayoría han visto pocos mensajes antitabaco que esto equivale a un 50.5\%, un $37.0 \%$ han visto muchos mensajes antitabaco en estos últimos días y el $12.5 \%$ que representa la minoría no ha visto ningún mensaje antitabaco últimamente.

\section{Conocimientos de los estudiantes del daño que ocasiona el hábito de fumar en la salud}

Casi el $100 \%$ de los encuestados opina que el humo de cigarrillo es dañino para la salud, siendo así se puede decir que concuerdan que ser fumadores pasivos (no fumadores) afectaría de alguna manera su salud. Acerca de la información que brinda la universidad sobre los daños que ocasiona el tabaco la mayor parte de las personas expresan que han recibido información muchas veces, seguida por las que refirieron haberla recibido en algún momento; finalmente un pequeño grupo de estudiantes refirió que nunca ha recibido información. 
De las personas encuestadas a quien se les pregunto si piensa que el tabaco es una droga la mayor parte refiere estar de acuerdo y un pequeño porcentaje no lo está. La aparición de cáncer pulmonar constituye la patología más conocida que aparece como consecuencia al consumo de cigarrillos, con un $32.5 \%$. Seguido a éste, las demás afecciones respiratorias (Aumento de la frecuencia de crisis de asma, tos, disnea, fatiga, infecciones, etc.) constituye la segunda respuesta más conocida, con un $17.5 \%$. El $11.6 \%$ tiene conocimiento que aparece un deterioro pulmonar en general. Por último, los problemas bucales/dentales obtuvieron un $9.1 \%$. Las demás respuestas obtuvieron porcentajes menores a éstos.

La mayoría de sexo femenino y masculino coincide en fumarse de 1 a 3 cigarrillos al día, seguido de 4 a 6 cigarrillos, en un tercer lugar de 7-9 cigarrillos en mujeres, pero de 10-12 cigarrillos en hombres y el cuarto lugar corresponde al mínimo porcentaje que fuma un paquete diario en ambos sexos. Con esto se puede observar que de las hombres y mujeres que fuman, no difieren en gran medida respecto a la cantidad de cigarrillos que fuman al día.

\section{Fuente y frecuencia de información en contra del consumo de tabaco}

De los encuestados la mayoría expresa que el medio que más implementa campañas antitabaco es la televisión, seguido por otras fuentes (carteles, revistas, películas. etc.) y en tercer lugar indican que ningún medio de comunicación implementa campañas en contra del tabaco. Del 100\% de total de los encuestados en su mayoría han visto pocos mensajes antitabaco que esto equivale a un $50.5 \%$, un $37.0 \%$ han visto muchos mensajes antitabaco en estos últimos días y el $12.5 \%$ que representa la minoría no ha visto ningún mensaje antitabaco últimamente.

\section{Valoración del conocimiento de los alumnos(as) sobre la Ley para el Control del Tabaco}

Seguidamente, los encuestados expresan saber que existe una ley que protege los derechos de los no fumadores y un porcentaje menor no está informado sobre este tema. Más de la mitad de los encuestados reflejaron que saben poco acerca de las prohibiciones y restricciones de la ley que protege a los no fumadores. Además, casi la totalidad de las personas desconocen quién es la autoridad competente para aplicar la ley para el control del tabaco. Únicamente una minoría desconoce acerca de la Declaración de la UNAN como Territorio Libre de Humo de Tabaco.

\section{CONCLUSIONES}

1. La prevalencia encontrada referente al consumo de cigarrillos en los estudiantes del primer a tercer año de la carrera de medicina de la UNAN-Managua del Recinto Universitario "Rubén Darío" es 17.1\%.

2. Según las variables socioeconómicas y demográficas, el índice de fumado en los hombres es mayor que en las mujeres.

3. Se pudo determinar que el consumo de cigarrillos es independiente de la cantidad de los ingresos económicos.

4. De los encuestados válidos reflejan que los lugares con mayor frecuencia en donde se aprende a fumar son el colegio, la universidad, casas de amigos y en fiestas y reuniones sociales. Minoritariamente en casa $\mathrm{y}$ en otros lugares.

5. Se observa que en los hogares en donde ambos padres fumaban, la prevalencia de hijos fumadores es mayor, en comparación con los hogares donde únicamente uno de los padres fumaba o en donde ninguno de ellos lo hacía. 
6. La convivencia con personas no pertenecientes al núcleo familiar incrementa la probabilidad de que una persona adquiera el hábito de consumir cigarrillos.

7. Por lo tanto se ha logrado destacar que la educación familiar ha tenido poca efectividad con respecto a la prevención de aparición de nuevos fumadores.

8. Se determinó que las personas que consideran que el hábito de consumir cigarrillos les facilitaría obtener más amistades, tienen mayor probabilidad de adquirir el hábito, al igual que las personas que sienten que fumar cigarrillos les incrementa su comodidad en reuniones sociales.

9. La aparición de cáncer pulmonar constituye la patología más conocida que aparece como consecuencia al consumo de cigarrillos.

10. Los encuestados refieren que las fuentes de donde obtienen mensajes en contra del consumo de tabaco provienen en mayor parte de la información brindada por la Universidad, la televisión, seguidos por otros como películas, revistas, carteleras, etc.

11. Más de la mitad de los encuestados reflejaron que saben poco acerca de las prohibiciones y restricciones de la ley que protege a los no fumadores. Además, casi la totalidad de las personas desconocen quién es la autoridad competente para aplicar la ley para el control del tabaco. Únicamente una minoría desconoce acerca de la Declaración de la UNAN como Territorio Libre de Humo de Tabaco.

\section{BIBLIOGRAFÍA}

Cabrera M. (2009). Hospital Universitario Quirón Madrid. Unidad de cardiología. Prevención, diagnóstico $y$ tratamiento de problemas cardiovasculares. http://www.cardioquiron.com/atencionalpaciente/tabaquismo.php

Consep, Sevip Ecuador. (1996). Segunda Encuesta Nacional Sobre Consumo de Drogas. Quito, Ecuador.

Consep. (1997).Primera Encuesta Nacional. Quito, Ecuador.

Cosen JN, de Cosen RH, Harper I. (2002). Tabaquismo; problemas éticos; consenso sobre contaminación del aire y tabaquismo. http://www.amamed.org.ar/publicaciones-revistas 1.asp? idrevista $=92$

De Santos Alba J. (2004). La influencia del tabaco en los tejidos bucales y su efecto sobre la cicatrización. http://www.ilustrados.com/publicaciones.

Encuesta de Tabaquismo en Jóvenes en Nicaragua, OPS/OMS. (2005).

García Ramos Alexis. (1993). Prácticas de alcoholismo y tabaquismo y relación con el rendimiento académico. (Monografía).

González, C. (2010). Factores de riesgo relacionados. Dieta y Tabaco. Centro de estudios Biosanitarios. http://eusalud.uninet.edu/misapuntes/index.php/Factores_de_riesgo_relacionados/

Pineda Whitaker Alice. (1994). Consumo de drogas ilegales (alcohol y tabaco) en escolares del instituto pedagógico La Salle. (Monografía). 
Sánchez, J. (2002) Material de Estadística Aplicada a la Educación. UNAN-Managua.

Susan, M. (1994) Estadística para Biología y Ciencias de la Salud. Interamericana-Mcgraw-Hill.

Valle Portilla MC del, Rodríguez Méndez CT, Llanes Llanes E. (2002). Guías prácticas clínicas. Enfermedades gingivales y periodontales. Ciudad de la Habana: http://aps.sld.cu/materiales/guías estomatológicas/cap4.pdf. 